Research Article

\title{
Readout Distance Enhancement of the Passive Wireless Multi-Parameter Sensing System Using a Repeater Coil
}

\author{
Lifeng Wang ${ }^{D}$, Lei Dong, and Qing-an Huang \\ Key Laboratory of MEMS of the Ministry of Education, Southeast University, Nanjing 210096, China \\ Correspondence should be addressed to Lifeng Wang; wanglifeng@seu.edu.cn
}

Received 24 October 2017; Accepted 10 January 2018; Published 12 February 2018

Academic Editor: Stephane Evoy

Copyright ( 2018 Lifeng Wang et al. This is an open access article distributed under the Creative Commons Attribution License, which permits unrestricted use, distribution, and reproduction in any medium, provided the original work is properly cited.

\begin{abstract}
A repeater coil is used to extend the detection distance of a passive wireless multi-parameter sensing system. The passive wireless sensing system has the ability of simultaneously monitoring three parameters by using backscatter modulation together with channel multiplexing. Two different repeater coils are designed and fabricated for readout distance enhancement of the sensing system: one is a PCB (printed circuit board) repeater coil, and the other is a copper wire repeater coil. Under the conditions of fixed voltage and adjustable voltage, the maximum readout distance of the sensing system with and without a repeater coil is measured. Experimental results show that larger power supply voltage can help further increase the readout distance. The maximum readout distance of the sensing system with a PCB repeater coil has been extended 2.3 times, and the one with a copper wire repeater coil has been extended 3 times. Theoretical analysis and experimental results both indicate that the high $Q$ factor repeater coil can extend the readout distance more. With the copper wire repeater coil as well as a higher power supply voltage, the passive wireless multi-parameter sensing system finally achieves a maximum readout distance of $13.5 \mathrm{~cm}$.
\end{abstract}

\section{Introduction}

Sensor technology is one of the most important technologies in the Internet of things [1]. However, in many application scenarios, such as human body, rotating parts, and harsh environments, the wire connections between the sensor and its circuit and/or between the sensor and its power supply cannot be allowed. Therefore, various passive wireless sensors have been designed and applied to these particular environments, most of which are based on the mechanism of inductive coupling [2-5].

One major drawback of inductive coupling is its short transmission distance. Generally, the transmission distance can only reach the length of the diameter of its inductor coil. With the miniaturization of the sizes of sensors, the readout distance of passive wireless sensors is becoming more and more prominent. The use of relay coils to increase transmission distance has been studied in wireless power transmission $[6,7]$. In terms of passive wireless sensors, a repeater has been used to increase the readout distance of an LC-type passive wireless sensor $[8,9]$. However, the added repeater brings the problems of frequency splitting and sensitivity reduction. Furthermore, the detection principle of LC sensor demands the resonant frequency of the repeater coil to be adjustable. As for these problems, a cyclic-scanning repeater is used to enhance the readout distance without frequency splitting and sensitivity reduction. And the cyclic-scanning repeater can cover the frequency range of the LC sensor [10]. Unfortunately, the cyclic-scanning repeater requires a wired power supply. In addition, the shortcoming of all these readoutenhanced LC-type sensors is that they only have the ability to measure single environmental parameter.

Based on our previous work [11], this paper uses a repeater coil to increase the readout distance of a passive wireless multi-parameter sensing system. The working principle in this paper is different from that in our previous articles $[9,10]$ : the former is based on the backscatter modulation while the latter is based on the LC resonant tank; so the repeater coil together with the sensing system needs to be redesigned. First of all, the sensing system is designed and simulated. Then, the design method of the repeater coil is given. After that, the sensing system without and with the 


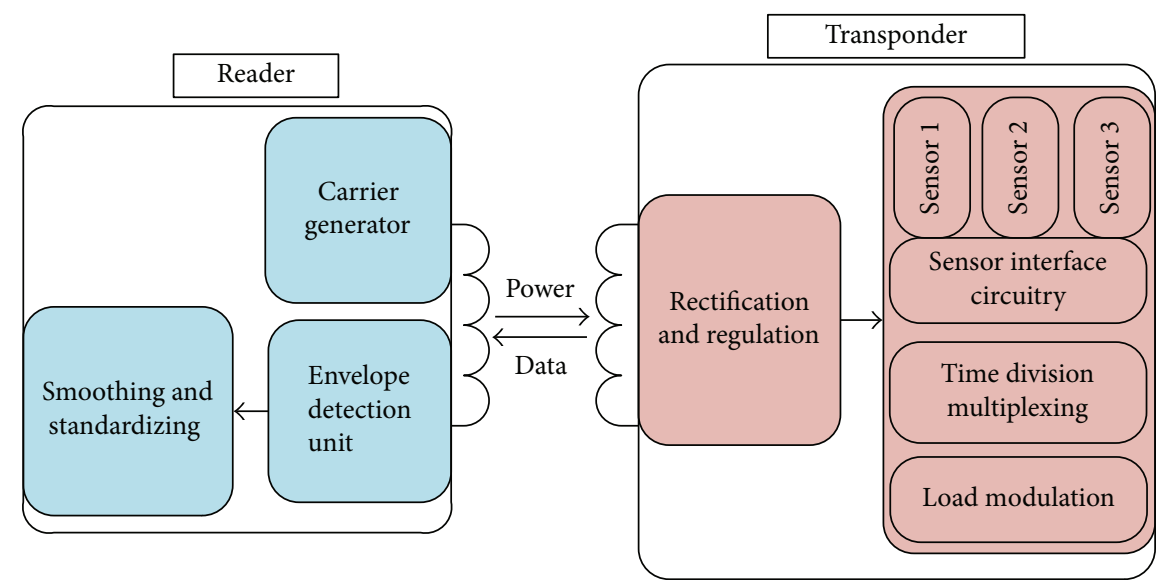

FIGURE 1: The overall block diagram of the passive wireless multi-parameter sensing system.

repeater coil is fabricated and tested, respectively. And a relatively long detection distance, multi-parameter, and passive wireless sensing system is established here.

\section{System Design}

For better understanding, the working principle of the passive wireless multi-parameter sensing system without the repeater is introduced first. Figure 1 shows the overall block diagram of the passive wireless multi-parameter sensing system. Basically, the system is composed of the transponder and the reader.

The passive wireless power and data transmission between the transponder and the reader are based on the inductive coupling mechanism. And the multi-parameter sensing of this system is realized by the backscatter modulation and the time division multiplexing. Firstly, the carrier generator unit in the reader generates a carrier signal, which is simultaneously coupled to the transponder through their inductor coils. Then, the rectification and regulation unit in the transponder rectifies and regulates the coupled carrier signal to a DC voltage. The three sensors together with their interfaces in the transponder can each generate a digital frequency signal, whose value is decided by the environment. Then, the time division multiplexer unit uses multiplex switches to sequentially transmit three digital frequency signals. After that, the load modulation circuit in the transponder modulates the digital frequency signals to the carrier signal. At the same time, the modulated carrier signal is coupled back to the reader. And in the reader, the envelope detection unit recovers the digital frequency signals from the modulated carrier signal. Finally, the demodulated digital frequency signals are smoothed and standardized.

According to the overall block diagram of the passive wireless multi-parameter sensing system in Figure 1, the reader circuit and the transponder circuit of the sensing system are designed in Multisim software [12] as shown in Figures 2(a) and 2(b), respectively. And the simulation results are shown in Figure 3. The digital frequency signals in the upper part of Figure 3 are the three sensors' signals and one synchronous signal in the transponder; and the digital frequency signals in the lower part are the demodulated and shaped signals in the reader. It means that the sensors' signals in the transponder are read out, passively and wirelessly, by the reader.

To increase the passive wireless detection distance of the sensing system above, a repeater coil is added. As shown in Figure 4, the repeater coil Lrp is placed at the middle of the transponder coil Ltr and the reader coil Lrd. In order to maximize the readout distance by the repeater coil, the following two conditions must be satisfied. The first is that the resonant frequency of the repeater coil should be the same as that of the reader coil and the transponder coil. And the second is that the reader coil, the transponder coil, and the repeater coil should all operate at their resonant states. When these two conditions are both satisfied at the same time, the strong magnetic coupling will occur and the readout distance will be significantly extended.

The maximum transfer distance between two magnetic coupling inductor coils is also related to several other factors, which are the sizes of the coils, the critical coupling coefficient, and the power supply. If the sizes of the coils are increased, the maximum transfer distance will be longer. Hence, the first way to increase the passive wireless transmission distance is to enlarge the sizes of the inductor coils. However, dimensions are usually restricted parameters. The critical coupling coefficient between two inductor coils could be defined as [9]

$$
k_{\mathrm{cr}}=\frac{1}{\sqrt{Q_{1} Q_{2}}},
$$

where $Q_{1}$ and $Q_{2}$ are the quality factors of the two inductor coils, respectively. When the coupling coefficient of the two inductor coils equals their critical coupling coefficient, it means that the passive wireless transmission efficiency between them reaches the highest. So the maximum transfer distance will be longer if the critical coupling coefficient is smaller. According to (1), the second way to increase the passive wireless transmission distance is to increase the $Q$ factor of the inductor coils. The relationship between the power supply and the readout distance is obvious. A larger 


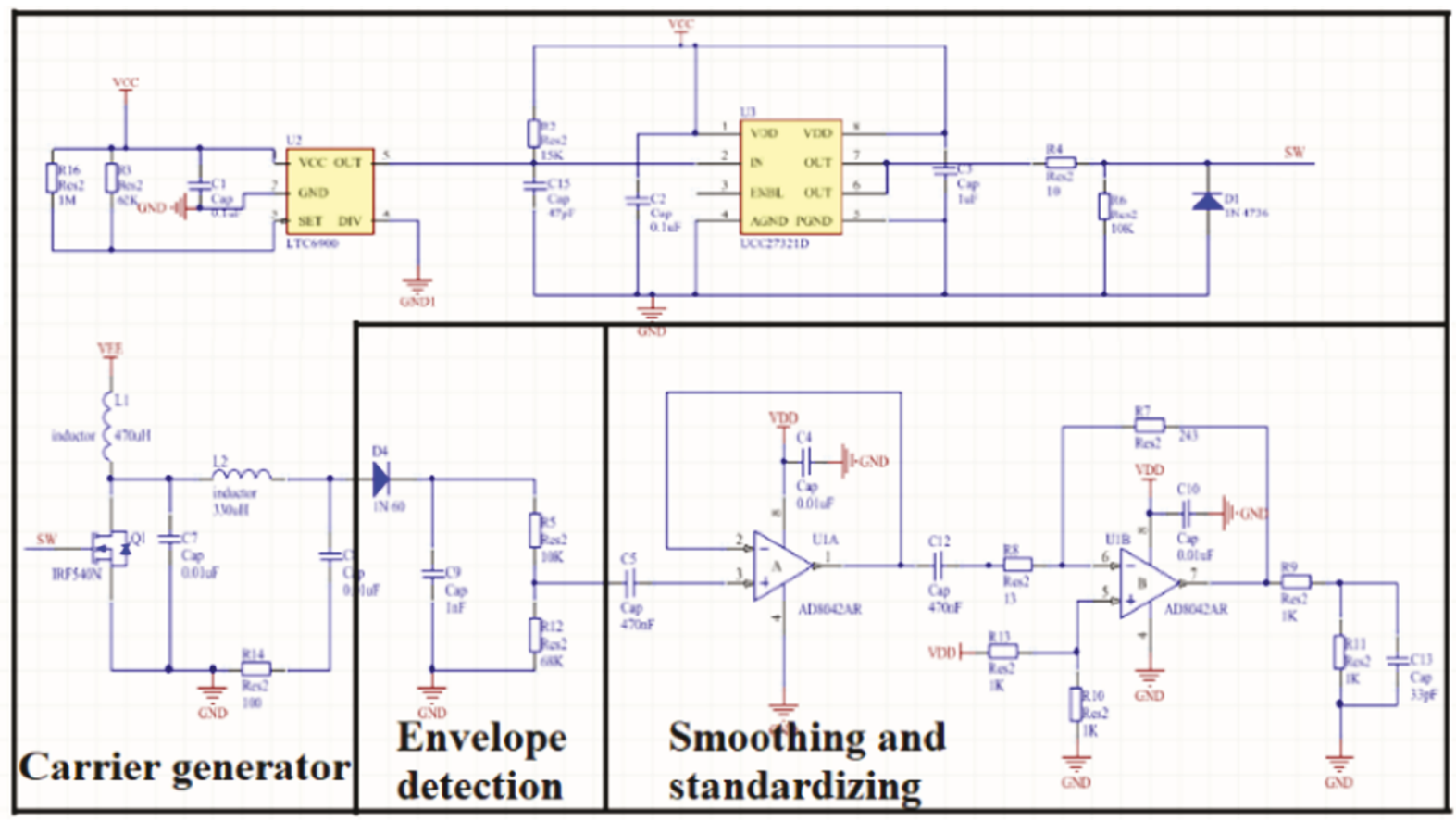

(a)

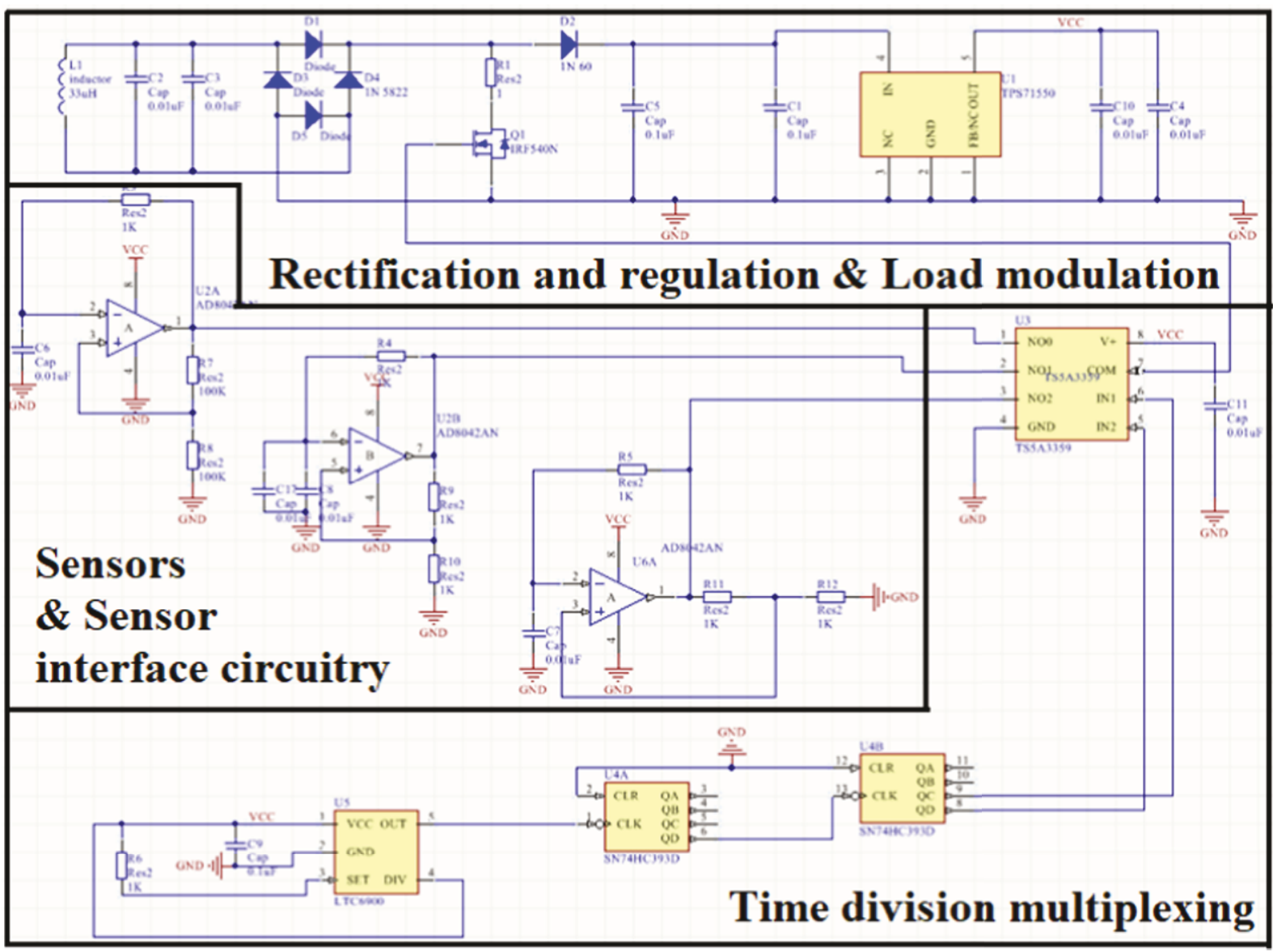

(b)

FIGURE 2: The sensing system designed in Multisim software: (a) the reader circuit and (b) the transponder circuit. 


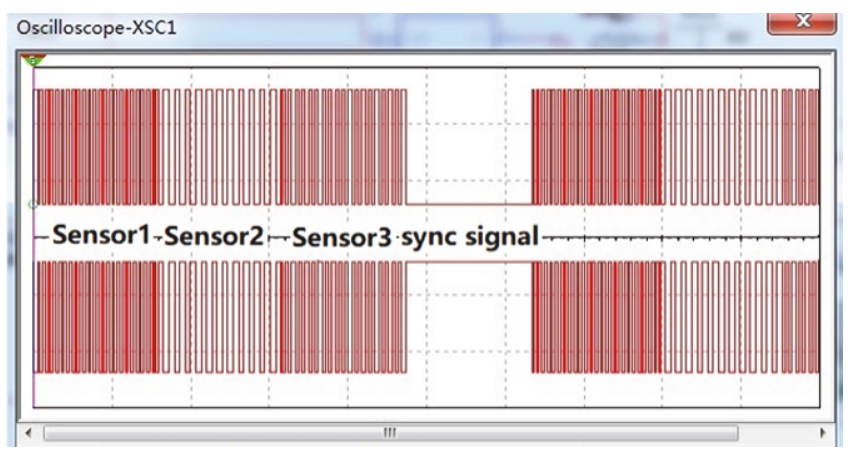

Figure 3: The simulation results of the passive wireless multiparameter sensing system. The digital frequency signals in the upper part are the three sensors' signals and one synchronous signal in the transponder; and the digital frequency signals in the lower part are the demodulated and shaped signals in the reader.

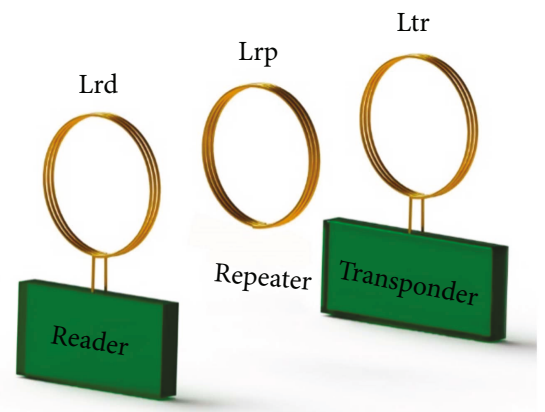

Figure 4: A repeater is added to increase the passive wireless detection distance of the sensing system.

power supply can generate stronger carrier signals, so the wireless transmission distance is longer. In another word, the third way to lengthen the readout distance is to increase the power supply.

\section{Experiments and Results}

According to the schematic in Figure 2, the transponder and the reader are fabricated based on PCBs (printed circuit boards). They have exactly the same size, that is, $5 \mathrm{~cm} \times 8.8 \mathrm{~cm}$. For a wireless transmission system, its bandwidth is proportional to its carrier frequency [13]. Therefore, the carrier frequency should be designed as high as possible. But the higher the carrier frequency is, the more difficult the design of the circuit will be. Based on compromise, the carrier frequency of our design is $5 \mathrm{MHz}$. And the measured value of the carrier frequency is $5.1 \mathrm{MHz}$. To make Lrd and Ltr both work at their resonant states, their resonant frequencies should be the same as the carrier frequency.

According to the carrier frequency, the two inductor coils, Lrd and Ltr, are designed based on the PCB process. They have exactly the same parameters: outer diameter $=4.4 \mathrm{~cm}$, turn $=8$, width of wire $=30 \mathrm{mil}$, and thickness of wire $=2 \mathrm{oz}$. After fabrication, the measured resonant frequencies of Lrd and Ltr are both $5.1 \mathrm{MHz}$.

Here, only the passive wireless readout distance between the reader and the transponder is going to be measured, so

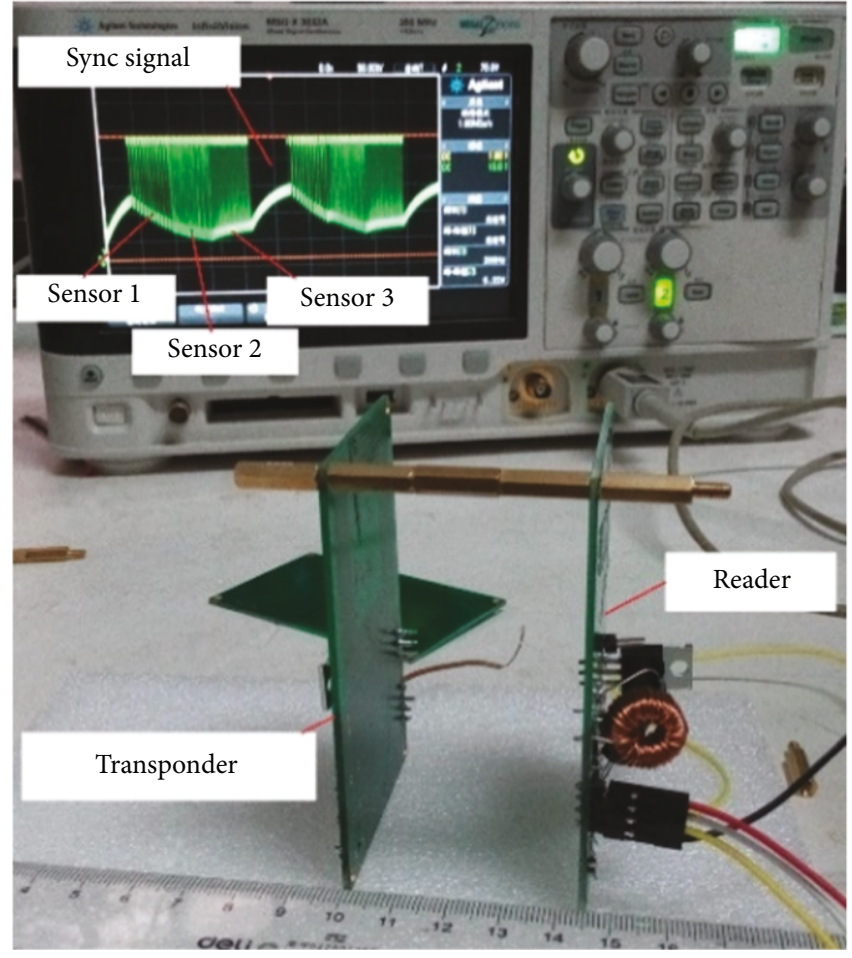

FIgURE 5: The measurement of the readout distance of the sensing system without a repeater coil.

three fixed value capacitors are used to represent the three capacitive sensors. Without and with the repeater coil, the passive wireless sensing system is tested, respectively.

3.1. Without a Repeater Coil. As shown in Figure 5, the readout distance of the sensing system without a repeater coil is measured. The reader coil and the transponder coil are face-to-face placed, and the output of the reader is observed by an oscilloscope (Agilent MSOX 3032A). The distance between the reader and the transponder is increased with the step of $1.5 \mathrm{~cm}$. Simultaneously, the power supply $V_{\mathrm{E}}$ of the reader is adjusted to ensure the output waveform of the reader is correct.

In Figure 5, the passive wireless readout distance between the transponder and the reader can be read as $4.5 \mathrm{~cm}$, which is the maximum readout distance without a repeater. That means, if the readout distance is increased, even if the power supply $V_{\mathrm{E}}$ is increased simultaneously, the correct output waveform will not be obtained. The minimum power supply $V_{\mathrm{E}}$ needed for the $4.5 \mathrm{~cm}$ readout distance is found as $9.1 \mathrm{~V}$.

On the screen, the three different digital frequency signals are corresponding to the three capacitive sensors in the transponder, respectively. The waveform displayed on the oscilloscope is a bit of distortion. This is because the distance between the two coils is too long, so that the voltage coupled to the transponder coil is close to or even lower than the critical operating voltage of the chips working on the transponder. Although this output waveform is slightly distorted compared to the simulation results in Figure 3, the frequencies of the three digital frequency signals can still be read correctly. 


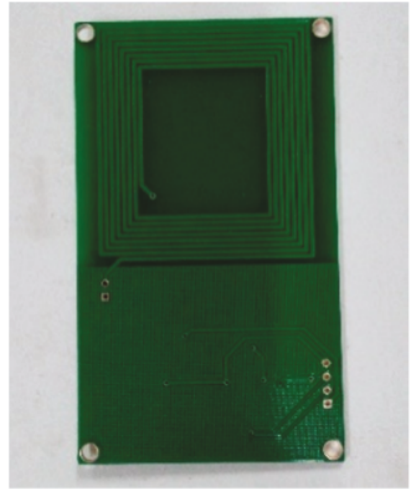

(a)

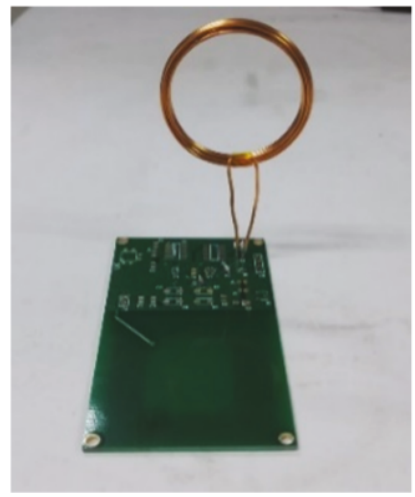

(b)

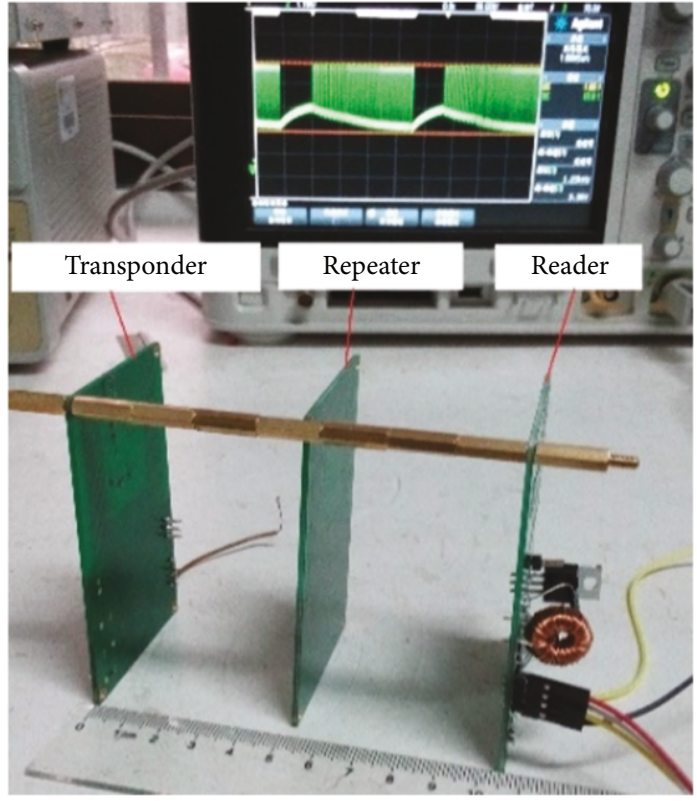

(c)

Figure 6: (a) The PCB repeater coil, (b) the copper wire repeater coil, and (c) the measurement of the readout distance of the sensing system with the PCB repeater coil.

3.2. With a Repeater Coil. According to the analysis in Section 2, the repeater coils we designed should meet the following two requirements. The first is that the resonant frequency of them should be the same as that of Lrd and Ltr; the second is that the size of them should be close to that of the reader and the transponder. Based on these two demands, two different repeater coils are prepared for readout distance measurement.

One repeater coil fabricated by PCB process is shown in Figure 6(a). The PCB repeater coil has exactly the same size as the transponder coil (or the reader coil). With identical size and fabrication process, the PCB repeater coils and the inductor coils on the reader and the transponder are easy to match into a strong magnetic coupling mode. In order to meet the requirement of strong magnetic coupling, the resonant frequency of the PCB repeater coil is tuned to $5.1 \mathrm{MHz}$ by connecting several capacitors (on the backside of the $\mathrm{PCB}$ ). And the measured $Q$ factor of the PCB repeater coil is 96 .

The other repeater coil, which is made of $1 \mathrm{~mm}$ copper wire, is shown in Figure 6(b). The copper wire coil chosen here is used to compare with the PCB coil, because the former often has higher $Q$ factor than the latter. Moreover, copper wire coils are easy making and common using inductor coils. The copper wire repeater coil in Figure 6(b) is $4 \mathrm{~cm}$ in diameter and has 6 turns. The copper wire repeater coil is connected to a PCB and is sustained by it. The resonant frequency of the copper wire repeater coil is adjusted to $5.1 \mathrm{MHz}$ too, and its measured $Q$ factor is 127 .

Then, the PCB repeater coil and the copper wire repeater coil are inserted between the transponder and the reader to extend the readout distance, respectively. Figure 6(c) shows the measurement setup of the passive wireless multiparameter sensing system with the PCB repeater coil. As shown in Figure 6(c), the maximum readout distance of the passive wireless sensing system reaches $10.5 \mathrm{~cm}$, while the output signals can still be read properly.

Actually, during the experiment, two ways are adopted to measure the passive wireless readout distance of the sensing system with a repeater coil. The first one is that the power supply $V_{\mathrm{E}}$ is fixed at $10 \mathrm{~V}$ to find out the maximum readout distance; the second one is that the power supply is allowed to be adjusted to get the maximum readout distance. Under the conditions of fixed voltage and adjustable voltage, the maximum readout distances of the sensing system without a repeater, with a PCB repeater coil, and with a copper wire repeater coil are listed in Table 1.

It can be seen from Table 1 that no matter the power supply is fixed or adjustable, the readout distance of the sensing system is greatly extended after a repeater coil is inserted in. Specifically, under fixed supply voltage, the readout distance of the sensing system with a PCB repeater coil has been extended 1.7 times, and the one with a copper wire repeater coil has been extended 2 times.

From the comparison between the fixed voltage and the adjustable voltage, it is obvious that increasing the voltage helps further increase the readout distance. Under adjustable supply voltage, the readout distance of the sensing system with a PCB repeater coil has been extended 2.3 times, and the one with a copper wire repeater coil has been extended 3 times. However, the supply voltage cannot be increased unlimitedly, because the chips on the reader have a certain upper limit of withstand voltage. Our circuit system cannot work steady at the power supply above $20 \mathrm{~V}$.

Furthermore, no matter the power supply is fixed or adjustable, the readout distance enhanced by the copper wire repeater coil is longer than that by the PCB repeater coil. In 
TABle 1: The maximum readout distances of the sensing system under different conditions.

\begin{tabular}{lcc}
\hline & $\begin{array}{c}\text { Power supply } \\
V_{\mathrm{E}}(\mathrm{V})\end{array}$ & $\begin{array}{c}\text { Readout } \\
\text { distance }(\mathrm{cm})\end{array}$ \\
\hline \multirow{2}{*}{ Without a repeater coil } & 10 & 4.5 \\
& 9.1 & 4.5 \\
\hline \multirow{2}{*}{ With a PCB repeater coil } & 10 & 7.5 \\
& 13.9 & 10.5 \\
\hline \multirow{2}{*}{ With a copper wire repeater coil } & 10 & 9 \\
& 19.3 & 13.5 \\
\hline
\end{tabular}

another word, the high- $Q$ repeater coil can extend the readout distance longer. Similarly, the bigger the better is not correct regarding the $Q$ factor of the repeater coils. Because the bandwidth of the wireless transmission system is inversely proportional to the $Q$ value of the inductor coils, the larger the $Q$ value is, the narrower the system bandwidth will be [13].

\section{Conclusion}

In the experiment, it is also found that the readout distance of the sensing system is the longest when the repeater coil is placed at the midpoint of the reader and the transponder. If the repeater coil is biased toward the reader or the transponder, the final readout distance of the sensing system will be smaller. Besides, the repeater coil must be coaxial with the reader coil and the transponder coil to maximize the readout distance of the system.

In summary, the detection distance of the passive wireless multi-parameter sensing system is greatly extended by using a repeater coil. With a larger power supply voltage, the maximum readout distance of the sensing system with the PCB repeater coil or with the copper wire repeater coil is 2.3 or 3 times as long as that without a repeater. And it is found that high- $Q$ repeater coil can extend the readout distance more. With the copper wire repeater coil, the passive wireless multi-parameter sensing system finally achieves a maximum readout distance of $13.5 \mathrm{~cm}$. This will help enlarge the application range of passive wireless sensors.

\section{Conflicts of Interest}

The authors declare that they have no conflicts of interest.

\section{Acknowledgments}

This work is supported by the National Natural Science Foundation of China (grant 61401084) and the National High Technology Development Program of China (grant 2015AA042602).

\section{References}

[1] L. Atzori, A. Iera, and G. Morabito, "The Internet of things: a survey," Computer Networks, vol. 54, no. 15, pp. 27872805, 2010.
[2] M. S. Mannoor, H. Tao, J. D. Clayton et al., "Graphene-based wireless bacteria detection on tooth enamel," Nature Communications, vol. 3, article 763, 2012.

[3] S. Bhadra, D. S. Y. Tan, D. J. Thomson, M. S. Freund, and G. E. Bridges, "A wireless passive sensor for temperature compensated remote $\mathrm{pH}$ monitoring," IEEE Sensors Journal, vol. 13, no. 6, pp. 2428-2436, 2013.

[4] M. G. Kisic, N. V. Blaz, K. B. Babkovic et al., "Passive wireless sensor for force measurements," IEEE Transactions on Magnetics, vol. 51, no. 1, pp. 1-4, 2015.

[5] Q. L. Tan, Z. Ren, T. Cai et al., "Wireless passive temperature sensor realized on multilayer HTCC tapes for harsh environment," Journal of Sensors, vol. 2015, Article ID 124058, 8 pages, 2015.

[6] A. Kurs, A. Karalis, R. Moffatt, J. D. Joannopoulos, P. Fisher, and M. Soljacic, "Wireless power transfer via strongly coupled magnetic resonances," Science, vol. 317, no. 5834, pp. 83-86, 2007.

[7] X. Wei, Z. Wang, and H. Dai, "A critical review of wireless power transfer via strongly coupled magnetic resonances," Energies, vol. 7, no. 7, pp. 4316-4341, 2014.

[8] D. A. Sanz, C. Mitrosbaras, E. A. Unigarro, and F. SeguraQuijano, "Passive resonators for wireless passive sensor readout enhancement," Applied Physics Letters, vol. 103, no. 13, article 133502, 2013.

[9] C. Zhang, L. F. Wang, and Q. A. Huang, "Extending the remote distance of LC passive wireless sensors via strongly coupled magnetic resonances," Journal of Micromechanics and Microengineering, vol. 24, no. 12, article 125021, 2014.

[10] L. Dong, L. F. Wang, C. Zhang, and Q. A. Huang, "A cyclic scanning repeater for enhancing the remote distance of LC passive wireless sensors," IEEE Transactions on Circuits and Systems I: Regular Papers, vol. 63, no. 9, pp. 1426-1433, 2016.

[11] L. Wang, J. Ma, Y. Huang, D. Tang, and Q. A. Huang, "Passive wireless hermetic environment monitoring system for spray painting workshop," Sensors, vol. 16, no. 8, article 1207, 2016.

[12] “Multisim.chm," February 2017, http://zone.ni.com/reference/ en-XX/help/375482B-01/.

[13] K. Finkenzeller, RFID Handbook, John Wiley \& Sons Ltd., Chichester, England, 1999. 


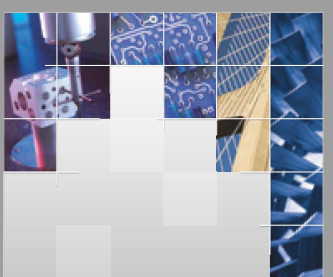

\section{Enfincering}
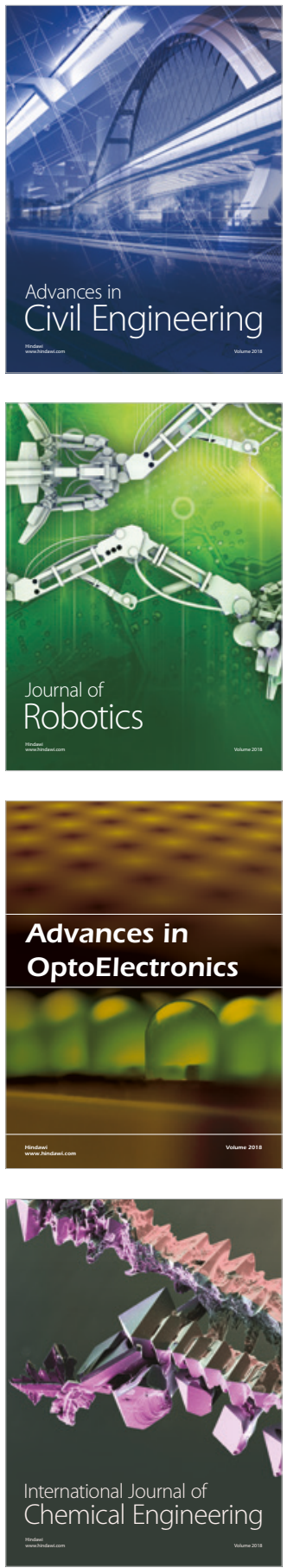

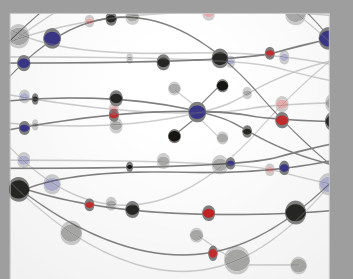

\section{Rotating \\ Machinery}

The Scientific World Journal

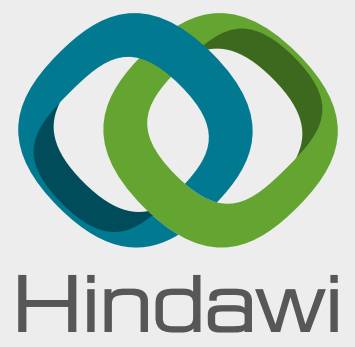

Submit your manuscripts at

www.hindawi.com
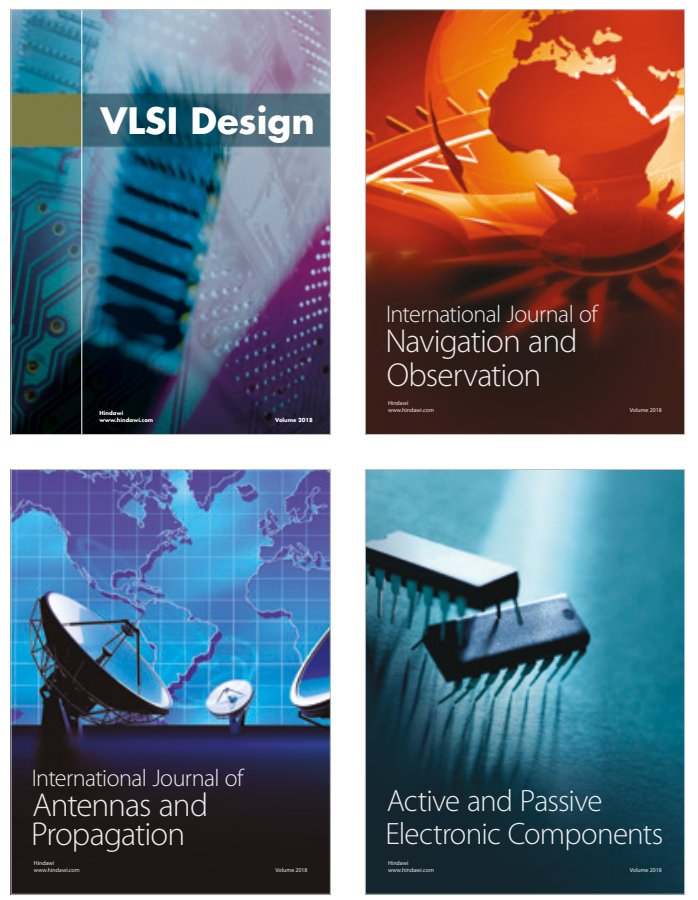
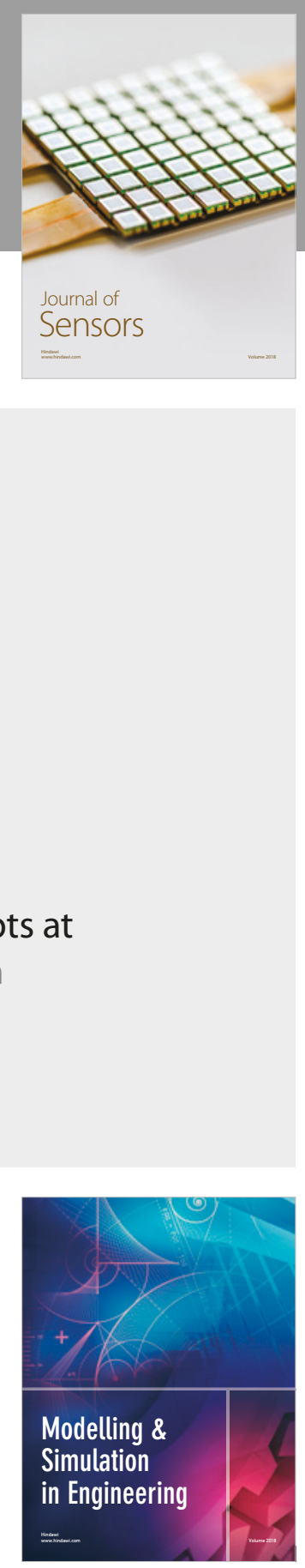

\section{Advances \\ Multimedia}
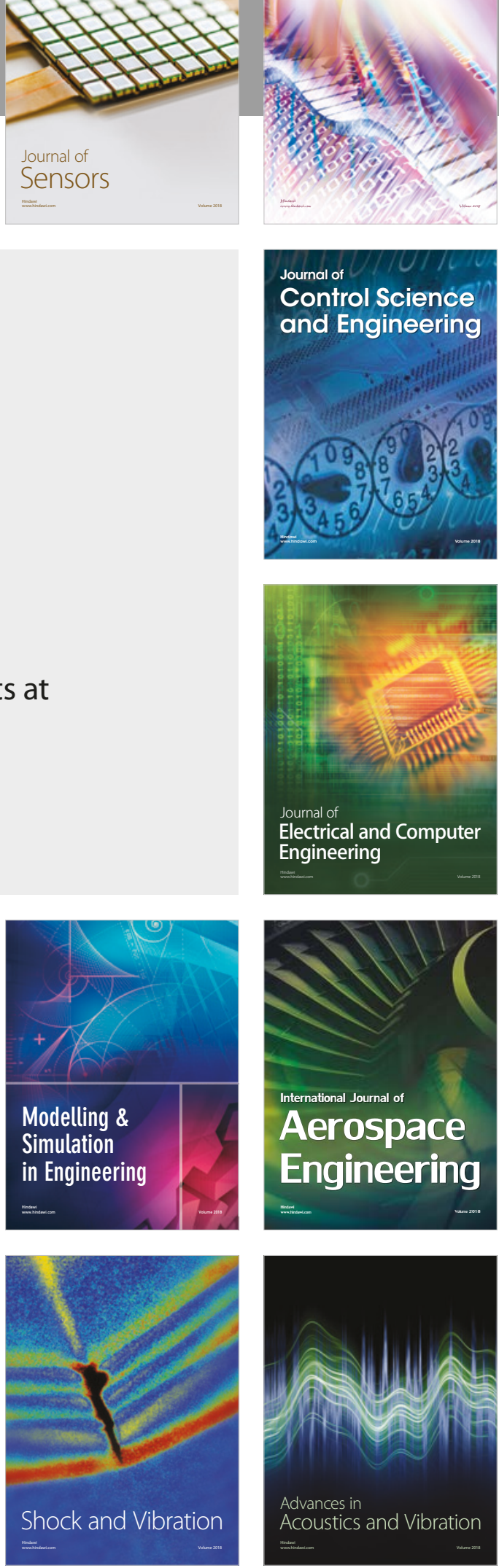